\title{
A Study of the Mechanical Characteristics of a Mandibular Parasymphyseal Fracture with Internal Fixation Device Subject to Variable Bite Forces: Finite Element Analysis
}

\author{
Victor Caraveo ${ }^{1}$, Scott Lovald ${ }^{2}$, Tariq Khraishi ${ }^{*}$ \\ ${ }^{1}$ Mechanical Engineering Department, University of New Mexico, Albuquerque, New Mexico, USA \\ ${ }^{2}$ Exponent, Inc., Menlo Park, CA, USA \\ Email: ${ }^{*}$ khraishi@unm.edu
}

How to cite this paper: Caraveo, V., Lovald, S. and Khraishi, T. (2021) A Study of the Mechanical Characteristics of a Mandibular Parasymphyseal Fracture with Internal Fixation Device Subject to Variable Bite Forces: Finite Element Analysis. Journal of Biosciences and Medicines, 9, 158-178. https://doi.org/10.4236/jbm.2021.94014

Received: March 1, 2021

Accepted: April 23, 2021

Published: April 26, 2021

Copyright $\odot 2021$ by author(s) and Scientific Research Publishing Inc. This work is licensed under the Creative Commons Attribution International License (CC BY 4.0).

http://creativecommons.org/licenses/by/4.0/

\begin{abstract}
In recent times, research into mandibular fracture has gained momentum from advances in scanning techniques, software/algorithm developments and improvements, and numerical structural modeling using the finite-element method (FEM). In this work, the FEM is used to model a mandibular fracture (using an inhomogeneous and orthotropic jaw model) simulating the effect of different bite tasks/forces on the stability of the fixated fracture. Specifically, bilateral and unilateral clenches (using muscle data) were studied using a low-profile 3D $4 \times 2$ hole mini-plate deployed for fracture fixation. Here, the mandible bone was treated as orthotropic and spatially inhomogeneous. Although the results of stress and displacement analyses, for this fixation hardware, indicate sufficient fixation under normal biting conditions, the results show that the unilateral and ipsilateral bites develop, in general, the highest stresses or displacements. Such results can guide post-surgery recommendation on bite behavior.
\end{abstract}

\section{Keywords}

Mandible, Mandibular Fracture, Internal Fixation, Finite-Element Modeling, Bite Forces

\section{Introduction}

A recent trend in researching complex modern problems has required interdisciplinary collaboration in order to develop solutions. This is the case in the problem facing maxillofacial surgeons who have relied heavily on trial and error 
methods for repairing mandibular fractures.

Open reduction and internal fixation is the most frequent treatment for mandibular fractures [1]. These procedures are largely considered successful, though studies have reported up to $30 \%$ rate of complications requiring plate removal (depending on the fracture site) [1] [2] [3] [4]. Methods outside the scope of the medical practitioner's expertise are necessary to understand the fundamental reasons by which a particular rigid internal fixation device may perform superior to another. Fixation of fractured mandibular bone segments is primarily of a structural nature involving various material properties, each exhibiting different mechanical responses (i.e. stress and strain under loading). The mechanical engineering discipline is suitable to further the efforts in developing improved plating systems and understanding the role of fracture mechanics in complications and removal after internal fixation.

A useful technique for analyzing complex structures is the finite element method (FEM), which essentially reduces a complex problem that might otherwise be impossible to solve analytically into numerous smaller and simpler problems generating a multitude of equations, or matrices, that can be solved numerically. Assembling each individual element matrix into the global stiffness matrix by applying the appropriate boundary conditions and reviewing the entire solution gives an understanding to the behavior of the structure. With the improvement in finite element analysis (FEA) software and the significant increase in capability of computing resources, FE modeling of biological tissues and physiological behavior is now becoming an established practice.

FEA offers insight into the mechanistic behavior of fixation plates used in rigid internal fixation and, if modeled carefully, could eventually become an accurate design tool. A literature investigation has sought to determine the appropriateness of fixation plating used in rigid internal fixation through FE analyses. Most studies have analyzed fixation by focusing on a single bite load, making it difficult to extrapolate findings to expected real world functioning. In addition, fracture contact conditions are largely ignored or greatly simplified. Surgeons will most often establish physical contact between the fractured mandibular bone pieces during surgery (reducing the fracture), allowing normal and frictional forces to mitigate some of the stresses and displacements that would otherwise be transferred entirely to the fixation hardware [5]. A study by Caraveo et al. [6] has determined the necessity to include frictional contact boundary conditions in the FEA analysis of the fracture region. Some of the studies that utilized one type of bite or bite task are [6] [7] [8].

One of the purposes of this investigation is to identify the effectiveness of a rigid internal fracture fixation device using FE simulations of the human mandible under variable bite forces. The primary bite forces are bilateral bite forces and unilateral bite forces both ipsilateral (interchangeably referred to the left side throughout this paper) and contralateral (right side) to the fracture site. A total of seven different bites and eleven different bite tasks are considered in this study. The authors hypothesize the biting tasks ipsilateral to the fracture will 
produce the greatest displacement in the fracture region.

Additionally, mechanical responses include stress measures in the plate-screw system as well as the cortical bone surrounding the screws. The commonly used von Mises criteria in FEA to evaluate mandibular bone stress studies is called into question since this measure is typically considered more suitable for ductile materials, whereas bone is characterized as having more of a brittle nature. The stress measure that captures the largest tensile value is the principal stress criteria in which the coordinate system is such that there are no shear stresses acting on the element and only normal stresses act in the three orthogonal directions. The $1^{\text {st }}$ principal stress is the largest of these three values, and if positive, indicates stress in tension. The peak compressive stress is indicated by the minimum principal stress value ( $3^{\text {rd }}$ principal stress), if it is negative. An additional goal of this study is to evaluate the differences in von Mises and principal stress components along the bone-implant interface.

\section{Methods}

\subsection{FEA Geometry Creation}

Computerized Tomography scans of a 22 year old male were obtained from a Siemens Somatone Sensations Multislice Scanner. The patient had full dentition and normal occlusion. The scans were imported into Mimics 7.3 (Materialise, Ann Arbor, MI) where thresholding and editing functions were used to create separate sections for cortical bone, cancellous bone, and the dental segment. IGES (Initial Graphics Exchange Specification) curves were approximated around the volumes and imported into ANSYS 8.0. Volumes were created and subsequently bonded in the symphysis, parasymphysis, body, angle, ramus, coronoid, and condyle regions using their respective IGES curves.

To mesh the volumes, tetrahedral-shaped solid elements were used, resulting in a large mesh totaling (for the mandible with the hardware) 65,927 elements and 101,115 nodes. Mesh convergence refinements of element sizes were conducted for the plate, screws, fracture region, and the surrounding cortical and cancellous regions. For the different biting scenarios, the same mesh was used to ensure consistency amongst FE studies. The actual 3D CAD model of the mandible was verified to match the original CT scans. Furthermore, loadings similar to previous studies were replicated in order to validate stress results [7] [8]. CAD model verification for this study was detailed in Chaudhary et al. [9] and Lovald et al. [7].

The fracture was simulated by dividing the mandibular corpus with a plane in the parasymphyseal region directly under the $1^{\text {st }}$ premolar as can be seen in Figure 1. Geometrical data for the plate was provided by the Stryker-Leibinger Corp (Kalamazoo, MI). The plate analyzed is the $3 \mathrm{D} 4 \times 2$ hole miniplate. This is a relatively flexible miniplate, with a $1 \mathrm{~mm}$ thickness. There is a small amount of clearance between the modeled plate and bone, as in clinical situations. Unicortical screw fixation was used on the superior border while bicortical fixation was 


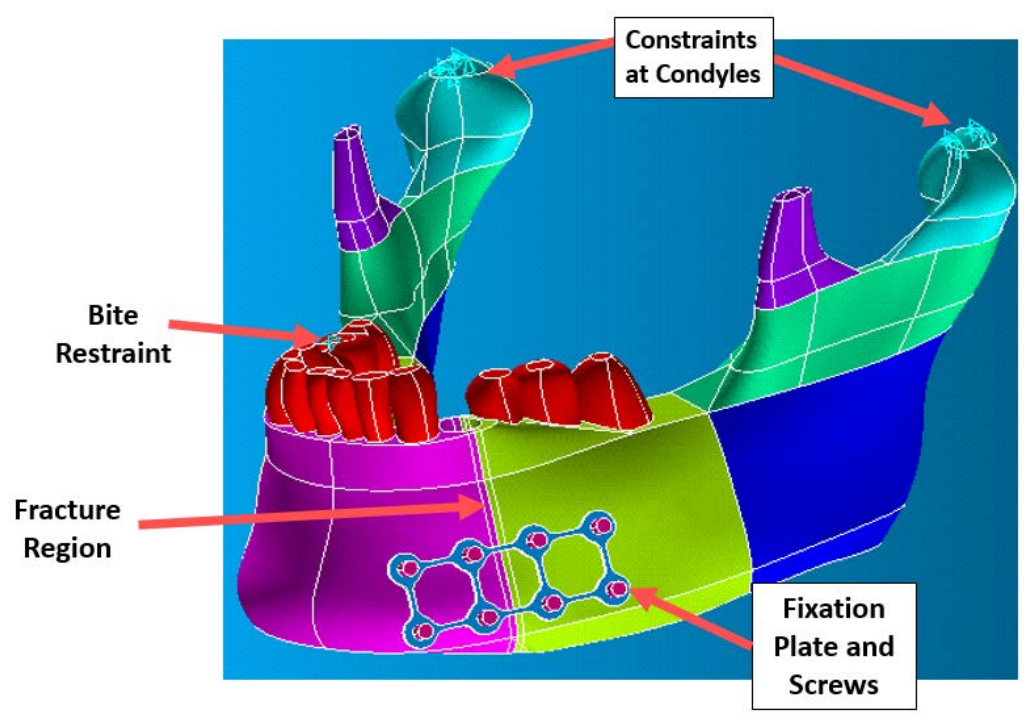

Figure 1. Mandible-fixation system geometry. Different colored regions represent different material property sections, i.e. inhomogeneous mandibular bone model.

used on the inferior border. Screws were simulated as solid cylinders with a diameter of $2.3 \mathrm{~mm}$ that were inserted and bonded to the bone material.

\subsection{Material Properties}

The mandible considered in this work consists of teeth, cortical bone, cancellous bone and dental segment. The dental segment consists of dentin, enamel, periodontal ligament. There are six symmetric volumes in the mandibular volume: the symphysis, parasymphysis, angle, ramus, condyle, and coronoid. That makes twelve the total number of mandibular volumes in the model. Each of these volumes was treated as orthotropic material, which is more accurate than the isotropic approximation. This required the specification of local coordinate systems into the FE software. Table 1 gives the material properties used in this study for simulating the fractured mandibular model. The orthotropic cortical bone values were taken from a study by Schwartz-Dabney et al. [10]. Isotropic properties for cancellous bone were taken from reference [11]. The properties for dentin are aligned with a study by Craig and Peyton [12]. Since dentin is much stiffer than other parts of the dental segment, its elastic properties were accounted for in the model. For the titanium plates, the properties were taken out of [13] which was a FE study of mandibular angle fractures. All bite load configurations utilized the same model; the only differences were the boundary condition and applied muscle load distribution corresponding to each respective bite task.

\subsection{Field Equations and Boundary Conditions}

Multiple bite forces were used in this FEA. Muscle force vectors that were experimentally derived for a specific bite are distributed around the mandible [14]. Each force has a direction, area of attachment, and magnitude. The condyles and occlusal surface at loading point are restrained from movement. 
Table 1. Material properties used in FEA of the mandible. Orthotropic properties were used for cortical bone, while isotropic properties were used for cancellous bone, dentin, and the titanium plate. The $x$-direction is along the length of the mandible, the $y$-direction is normal to the bone surface, and the $\mathrm{z}$-direction is their cross product.

\begin{tabular}{cccccccccc}
\hline Material Properties & Symphysis & Parasymphysis & Angle & Ramus & Condyle & Coronoid & Cancellous & Dentin & Titanium \\
\hline$E_{x}$ & 20,492 & 21,728 & 23,793 & 24,607 & 23,500 & 28,000 & 7930 & 17,600 & 110,000 \\
$E_{y}$ & 12,092 & 12,700 & 12,757 & 12,971 & 12,650 & 14,000 & 7930 & 17,600 & 110,000 \\
$E_{z}$ & 16,350 & 17,828 & 19,014 & 18,357 & 17,850 & 17,500 & 7930 & 17,600 & 110,000 \\
$\boldsymbol{v}_{x y}$ & 0.43 & 0.45 & 0.41 & 0.38 & 0.32 & 0.28 & 0.3 & 0.34 & 0.34 \\
$\boldsymbol{v}_{y z}$ & 0.22 & 0.2 & 0.22 & 0.23 & 0.25 & 0.28 & 0.3 & 0.34 & 0.34 \\
$\boldsymbol{v}_{x z}$ & 0.34 & 0.34 & 0.3 & 0.28 & 0.24 & 0.23 & 0.3 & 0.34 & 0.34 \\
$G_{x y}$ & 5317 & 5533 & 5493 & 5386 & 5500 & 5750 & 3050 & 6567 & 41,045 \\
$G_{y z}$ & 4825 & 5083 & 4986 & 5014 & 5150 & 5300 & 3050 & 6567 & 41,045 \\
$G_{x z}$ & 6908 & 7450 & 7579 & 7407 & 7150 & 7150 & 3050 & 6567 & 41,045 \\
\hline
\end{tabular}

The equations of equilibrium used in this mandibular FEA are:

$$
\sigma_{i j, j}+f_{i}=0, i=1,2,3, j=1,2,3
$$

In the realm of small deformation theory, the strain $\varepsilon_{i j}$ at a material point is related to the gradients of displacement as follows:

$$
\varepsilon_{i j}=\frac{1}{2}\left(u_{i, j}+u_{j, i}\right), i=1,2,3, j=1,2,3
$$

For an orthotropic material deforming elastically, the stress-strain constitutive relation, or Hooke's Law, is as follows:

$$
\begin{aligned}
& \varepsilon_{i i}=\frac{1}{E_{i}} \sigma_{i i}-\frac{v_{j i}}{E_{j}} \sigma_{j j}-\frac{v_{k i}}{E_{k}} \sigma_{k k}, i=1,2,3, j=1,2,3, k=1,2,3, i \neq j \neq k \\
& \varepsilon_{i j}=\frac{1}{2 G_{i j}} \sigma_{i j}, i=1,2,3, j=1,2,3, i \neq j
\end{aligned}
$$

where $E_{i}, G_{i p}$ and $v_{i j}$ are the different elastic material constants ( 9 total). In this last equation, a repeated index does not imply summation.

For an isotropic material, Hooke's Law reduces to two material constants (two from $G, \lambda$ and $v)$ :

$$
\varepsilon_{i j}=\frac{1}{2 G}\left(\sigma_{i j}-\frac{\lambda \delta_{i j}}{2 G+3 \lambda} \sigma_{k k}\right), i=1,2,3, j=1,2,3, k=1,2,3
$$

where

$$
\lambda=\frac{E v}{(1+v)(1-2 v)}
$$

Equations (1), (2), (3) and (4) apply to material elements in the whole mandibular domain, $\Omega$. Here $\sigma_{i j}$ is the $i j$ th component of the stress tensor, and $f_{i}$ is the $i$ th component of acting body forces. Body force contribution is negligible in this case.

The boundary conditions used in this mandibular FEA are: 


$$
\begin{gathered}
u_{i}=0, i=1,2,3 \\
\sigma_{i j} n_{j}=T_{i}, i=1,2,3, j=1,2,3
\end{gathered}
$$

$u_{i}$ in Equation (5) is the $i$ th component of the displacement vector of a material point. Equation (6) represents the stress traction, or force per area, exerted by muscle forces during mastication on the mandible. Here $n_{j}$ is the $j$ th component of the normal unit vector at a surface point $\mathrm{P}$ and $T_{i}$ is the $i$ th component of traction. The condition of zero displacement in Equation (5) applies only to the condyle surfaces $\left(\Gamma_{C}\right)$ and the occlusal surface of the tooth where the mastication takes place $\left(\Gamma_{T}\right)$; an example is illustrated in Figure 1.

\subsubsection{Frictional Contact Boundary Conditions}

Frictional contact boundary elements were applied to the surfaces of the fracture. Double-sided surface-to-surface contact boundary elements were utilized since it was not known initially which surface initiated contact with the other surface for each load configuration. The same coefficient of friction utilized in a previous study by the authors $(\mu=0.4)[6]$, and obtained from an experimental study [15], is used for this model. ANSYS contact elements used in the ANSYS FE models were TARGE170 and CONTA174 (Figure 2 shows these elements for a cross-section of the mandible, specifically the cortical bone, representing one of the fracture surfaces).

The algorithm chosen for the contact elements was the Augmented Lagrangian Method which requires more iterations than the Pure Penalty Method but was chosen for better conditioning in the event of element distortion. For contact analysis, the traction vector for contact has three components: one normal

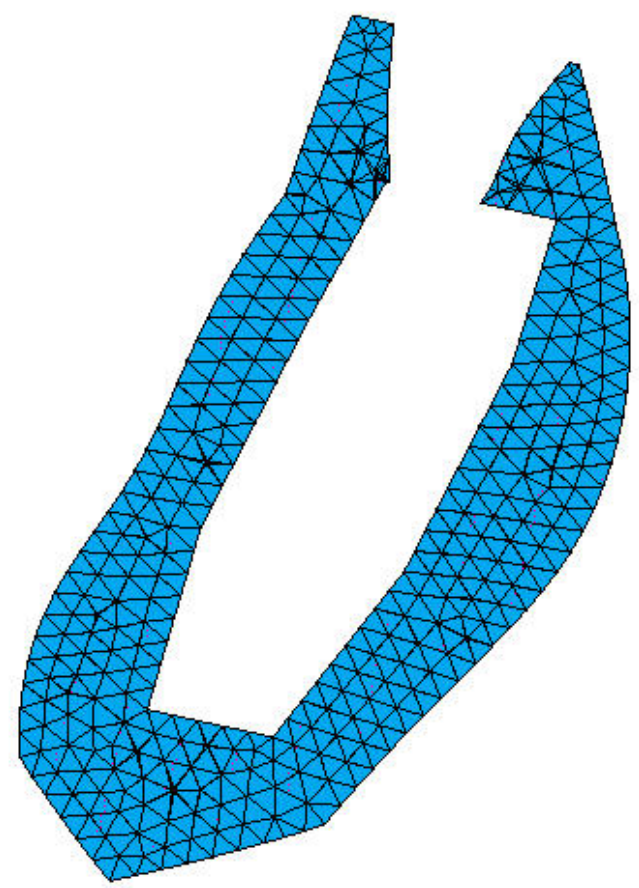

Figure 2. Cross section view of fracture depicting triangular contact elements. 
(Pressure or $\mathrm{P}$ ) and two tangential (frictional) shear stresses (in the local $y$ and $z$ directions here, i.e. $\tau_{y}$ and $\tau_{z}$ ). The contact pressure is defined using Lagrange multiplier as it is directly related to the contact normal stiffness $\left(K_{n}\right)$ and the contact gap size $\left(u_{n}\right)$. The Lagrange multiplier at iteration $i\left(\lambda_{i}\right)$ is computed locally (for each element) and iteratively. The frictional stress is obtained by Coulomb's law:

$$
\tau_{i}=\left\{\begin{array}{cc}
\tau_{i}^{n-1}+K_{s} \Delta u_{i} & \text { if } \tau=\sqrt{\tau_{y}^{2}+\tau_{z}^{2}}-\mu P<0 \text { (sticking) } \\
\mu P \frac{\Delta u_{i}}{\Delta u} & \text { if } \tau=\sqrt{\tau_{y}^{2}+\tau_{z}^{2}}-\mu P=0 \text { (sliding) }
\end{array}\right.
$$

where $\tau_{i}^{n-1}$ is the frictional stress in direction $i=1,2$ at the end of the previous substep, $\tau$ is the equivalent stress, $K_{s}$ is the tangential contact stiffness, $\Delta u_{i}$ is the slip increment in direction $i$ over the current substep, $\Delta u$ is the equivalent slip increment over the current substep, and $\mu$ is the coefficient of friction. The last equation is obtained from an ANSYS manual.

\subsubsection{Mastication Forces}

The magnitude and direction of muscle forces during the simulated bite were obtained from Nelson et al. [16]. Nelson's work is seen as an authority in muscle data forces and is the standard in most literature that have attempted to simulate bite forces in previous FEA studies [6] [7] [8] [14]. The data from this reference pertains to the bite of a healthy adult with an intact mandible. It is estimated that the bite force of a patient with a fractured mandible is $60 \%$ of that of a healthy adult [17]. The bite force data was modified accordingly in this study. The muscle attachment areas on the mandible were obtained from the literature [18].

Muscle forces in the FE model adapted muscle force data from previous studies [14] [16] and include nine bilateral muscles: superior masseter, deep masseter, medial pterygoid, anterior temporalis, middle temporalis, posterior temporalis, inferior lateral pterygoid, superior lateral pterygoid, and anterior digastrics. The resultant muscle forces are described by the force vector and a central muscle insertion point. These direction cosines describing the direction of muscle forces are given in Table 2. The magnitude of each resultant muscle force is given by:

$$
\left[X_{M i} \cdot K\right] \cdot E M G_{M i}=M_{i r}
$$

$X_{M i}$ is the cross sectional area of the muscle attachment in $\mathrm{cm}^{2}$ and $K$ is a general conversion constant for skeletal muscle expressed in $\mathrm{N} / \mathrm{cm}^{2}$. The product $\left[X_{M i} \cdot K\right]$ is known as the Weighting Factor $(N)$ and gives the maximum possible muscle force for a muscle. The term $E M G_{M i}$ is a scaling factor for each biting task that scales down the muscle force in proportion to the maximum possible muscle force exerted by a particular muscle group. The product $\left(M_{i r}\right)$ given by Equation (8) is the magnitude of the muscle force for a particular muscle. These forces were further distributed to the nodes attached to each muscle attachment area in the model. There are two major types of bite forces that have been analyzed 
Table 2. Muscle weighing factors, $\left[X_{M i} \cdot K\right]$, for each masticatory muscle group and direction cosines of muscle direction components where the $x$ - $Z$ plane is parallel to occlusal plane and $y-z$ plane is the sagittal plane perpendicular to the occlusal plane. Muscle force data was adapted from the literature [14] [16].

\begin{tabular}{cccccccc}
\hline & $\begin{array}{c}\text { Weighting } \\
\text { Factor (N) }\end{array}$ & \multicolumn{3}{c}{$\begin{array}{c}\text { Right Side } \\
\text { Direction Cosines }\end{array}$} & \multicolumn{2}{c}{ Left Side Direction Cosines } \\
& & cos- $X$ & cos- $y$ & $\cos -Z$ & $\cos -X$ & $\cos -y$ & $\cos -Z$ \\
\hline Superficial Masseter & 190.4 & -0.207 & 0.884 & 0.419 & 0.207 & 0.884 & 0.419 \\
Deep Masseter & 81.6 & -0.546 & 0.758 & -0.358 & 0.546 & 0.758 & -0.358 \\
Medial pterygoid & 174.8 & 0.486 & 0.791 & 0.372 & -0.486 & 0.791 & 0.372 \\
Anterior temporalis & 158 & -0.149 & 0.988 & 0.044 & 0.149 & 0.988 & 0.044 \\
Middle Temporalis & 95.6 & -0.221 & 0.837 & -0.5 & 0.221 & 0.837 & -0.5 \\
Posterior Temporalis & 75.6 & -0.208 & 0.474 & -0.855 & 0.208 & 0.474 & -0.855 \\
Inferior lateral pterygoid & 66.9 & 0.63 & -0.174 & 0.757 & -0.63 & -0.174 & 0.757 \\
Superior lateral pterygoid & 28.7 & 0.761 & 0.074 & 0.645 & -0.761 & 0.074 & 0.645 \\
Anterior Digastrics & 40 & -0.244 & -0.237 & -0.94 & 0.244 & -0.237 & -0.244 \\
\hline
\end{tabular}

in this study; symmetrical bilateral clenching and unsymmetrical unilateral bite forces, both ipsilateral and contralateral to the fracture region.

The three symmetrical bilateral clenching tasks are: incisal (I), intercuspal (IC), and the bilateral molar clenches (BM). The incisal clench involves an anterior clenching emphasis and is constrained at both incisors in the tooth appropriate tooth reaction direction. The intercuspal clench is medially concentrated in the mandible and restraints are applied at two bilateral tooth locations ( $1^{\text {st }}$ premolar and $1^{\text {st }}$ molar). Bilateral molar clenching tasks concentrate forces in the posterior occlusal region and are restrained in the $1^{\text {st }}$ and $2^{\text {nd }}$ molars for each analysis.

Asymmetrical unilateral clenching tasks simulate bite loading that consists of working side muscles and balancing side muscles [16]. Each mastication task is evaluated both ipsilateral and contralateral to the fracture site. The unilateral clenching tasks include the right and left side unilateral canine clench (LUC and RUC) restrained at each respective canine. The unilateral molar clench is configured for the left (LUM) and right (RUM) side as well as being constrained at both the $1^{\text {st }}$ and $2^{\text {nd }}$ molars making for a total of four unilateral molar clenching tasks. The scaling factors, $\mathrm{EMG}_{\mathrm{Mi}}$, for masticatory muscle forces for each of the bilateral clenching tasks, and the unilateral clenching tasks, are listed in Table 3 and Table 4.

\section{Results}

\subsection{Stress Results}

\subsubsection{Cortical Bone Stress - Von Mises, 1st Principal, and 3rd Principal Stress}

Three measures of interest are the maximum nodal Von Mises stress, the $1^{\text {st }}$ 
Table 3. Bilateral clenching left and right side muscle scale factors, $\mathrm{EMG}_{\mathrm{Mi}}$.

\begin{tabular}{ccccccc}
\hline & \multicolumn{5}{c}{ Bilateral Clenching } \\
\cline { 2 - 7 } Mastication Muscles & \multicolumn{2}{c}{ Intercuspal } & \multicolumn{2}{c}{ Bilateral } & \multicolumn{2}{c}{ Incisal } \\
\cline { 2 - 8 } & $\mathrm{R}$ & $\mathrm{L}$ & $\mathrm{R}$ & $\mathrm{L}$ & $\mathrm{R}$ & $\mathrm{L}$ \\
\hline Superficial Masseter & 1 & 1 & 0.81 & 0.81 & 0.4 & 0.4 \\
Deep Masseter & 1 & 1 & 0.81 & 0.81 & 0.26 & 0.26 \\
Medial pterygoid & 0.76 & 0.76 & 0.82 & 0.82 & 0.78 & 0.78 \\
Anterior temporalis & 0.98 & 0.98 & 0.83 & 0.83 & 0.08 & 0.08 \\
Middle Temporalis & 0.96 & 0.96 & 0.83 & 0.83 & 0.06 & 0.06 \\
Posterior Temporalis & 0.94 & 0.94 & 0.84 & 0.84 & 0.04 & 0.04 \\
Inferior lateral pterygoid & 0.27 & 0.27 & 0.36 & 0.36 & 0.71 & 0.71 \\
Superior lateral pterygoid & 0.59 & 0.59 & 0.61 & 0.61 & 0.5 & 0.5 \\
Anterior Digastrics & 0.28 & 0.28 & 0.33 & 0.33 & 0.5 & 0.5 \\
\hline
\end{tabular}

Table 4. Unilateral clenching left and right side muscle scale factors, $\mathrm{EMG}_{\mathrm{Mi}}$.

\begin{tabular}{cccccccccc}
\hline & \multicolumn{8}{c}{ Unilateral Clenching } \\
\cline { 2 - 10 } Mastication Muscles & \multicolumn{2}{c}{ RUM } & \multicolumn{2}{c}{ LUM } & \multicolumn{2}{c}{ RUC } & \multicolumn{2}{c}{ LUC } \\
\cline { 2 - 10 } & $\mathrm{R}$ & $\mathrm{L}$ & $\mathrm{R}$ & $\mathrm{L}$ & $\mathrm{R}$ & $\mathrm{L}$ & $\mathrm{R}$ & $\mathrm{L}$ \\
\hline Superficial Masseter & 0.72 & 0.6 & 0.6 & 0.72 & 0.46 & 0.58 & 0.58 & 0.46 \\
Deep Masseter & 0.72 & 0.6 & 0.6 & 0.72 & 0.46 & 0.58 & 0.58 & 0.46 \\
Medial pterygoid & 0.84 & 0.6 & 0.6 & 0.84 & 0.55 & 0.47 & 0.47 & 0.55 \\
Anterior temporalis & 0.73 & 0.58 & 0.58 & 0.73 & 0.54 & 0.14 & 0.14 & 0.54 \\
Middle Temporalis & 0.66 & 0.67 & 0.67 & 0.66 & 0.48 & 0.2 & 0.2 & 0.48 \\
Posterior Temporalis & 0.59 & 0.39 & 0.39 & 0.59 & 0.42 & 0.26 & 0.26 & 0.42 \\
Inferior lateral pterygoid & 0.14 & 0.59 & 0.59 & 0.14 & 0.3 & 0.65 & 0.65 & 0.3 \\
Superior lateral pterygoid & 0 & 0 & 0 & 0 & 0 & 0 & 0 & 0 \\
Anterior Digastrics & 0 & 0 & 0 & 0 & 0 & 0 & 0 & 0 \\
\hline
\end{tabular}

principal stress and the minimum $3^{\text {rd }}$ principal stress values for the cortical bone in all biting tasks. In regards to principal stress, positive values represent tension while negative values represent compression. Von mises stress is always a scalar positive value that represents the general magnitude of stress at a point. The FEA model results for bone stress are recorded in Table 5.

For illustrative purposes, contour plots of the three stress measures of interest for an incisal clench (INC) and the left unilateral molar clench restrained at the first molar (LUM1) are shown in Figure 3. For the incisal biting task, the stress distribution contours appear to be very similar, however, the maximum $1^{\text {st }}$ principal stress location is at the anterior (with respect to fracture plane) bone segment surrounding the superior screw hole proximal to the fracture, while the maximum Von Mises Stress value can be found in the posterior bone section 
Table 5. Bone stress-peak von mises, $1^{\text {st }}$ Principal Stress, and $3^{\text {rd }}$ principal stress comparison for all biting tasks. Note: BM1: bilateral $1^{\text {st }}$ molar clench , BM2: bilateral $2^{\text {nd }}$ molar clench, ICPM: intercuspal clench at 2 nd premolar IC1M: intercuspal clench at $1^{\text {st }}$ molar, INC: Incisal, RUC: right unilateral canine clench, LUC: left unilateral canine clench, LUM1: left unilateral molar clench restrained at the 1st molar, LUM2: left unilateral molar clench restrained at the 2nd molar, RUM1: right unilateral molar clench restrained at the 1st molar, RUM2: right unilateral molar clench restrained at the $2^{\text {nd }}$ molar.

\begin{tabular}{cccccc}
\hline Bite Task & $\begin{array}{c}\text { Max Nodal Von } \\
\text { Mises Stress }\end{array}$ & $\begin{array}{c}\text { Max 1st } \\
\text { Principal Stress }\end{array}$ & $\begin{array}{c}\text { \% Von Mises } \\
\text { difference }\end{array}$ & $\begin{array}{c}\text { Min 3rd } \\
\text { principal stress }\end{array}$ & $\begin{array}{c}\text { \% Von Mises } \\
\text { difference }\end{array}$ \\
\hline BM1 & 25.2 & 21.7 & $13.8 \%$ & -27.8 & $10.3 \%$ \\
BM2 & 27.0 & 21.2 & $21.7 \%$ & -28.7 & $6.2 \%$ \\
ICPM & 27.7 & 26.1 & $5.7 \%$ & -30.4 & $9.9 \%$ \\
IC1M & 33.3 & 29.0 & $12.8 \%$ & -36.5 & $9.8 \%$ \\
INC & 60.0 & 62.5 & $4.2 \%$ & -67.4 & $12.4 \%$ \\
RUC & 34.7 & 44.4 & $27.7 \%$ & -28.9 & $16.8 \%$ \\
LUC & 42.8 & 48.2 & $12.6 \%$ & -37.0 & $13.5 \%$ \\
LUM1 & 85.5 & 64.1 & $25.0 \%$ & -103.9 & $21.6 \%$ \\
LUM2 & 86.3 & 63.8 & $26.0 \%$ & -104.9 & $21.6 \%$ \\
RUM1 & 57.6 & 73.2 & $27.0 \%$ & -47.7 & $17.2 \%$ \\
RUM2 & 52.1 & 66.3 & $27.3 \%$ & -43.3 & 16.8 \\
\hline
\end{tabular}
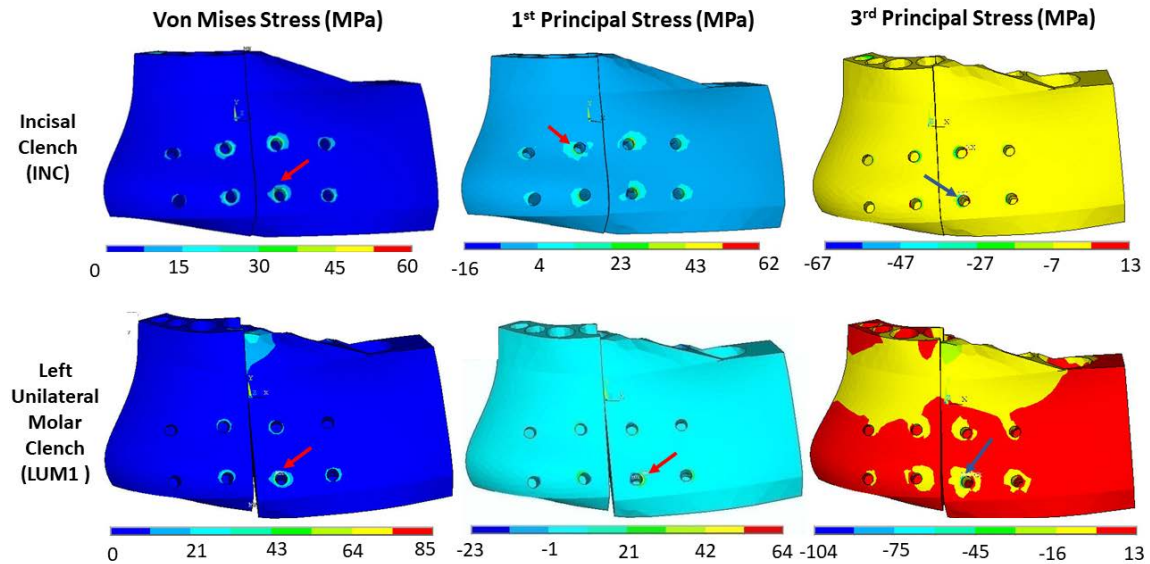

Figure 3. Cortical bone stress contour plots (MPa) for the incisal clench (INC) and the left unilateral molar clench (LUM1). Red arrows indicate the maximum stress location. Blue arrows indicate the minimum stress location ( $3^{\text {rd }}$ Principal Stress).

surrounding the proximal inferior screw hole (indicated with red arrow). The minimum $3^{\text {rd }}$ principal stress value is also located at the postero-inferior screw location. There is a $4.2 \%$ difference in peak stress values for the $1^{\text {st }}$ principal stress from the Von Mises stress and a $12.4 \%$ difference for the $3^{\text {rd }}$ principal stress for this bite task. Not that peak stress values in cortical bone in this study should be interpreted in a relative fashion due to simplifications to the screw geometry and the interface conditions between the screw bodies and the bone.

For the left unilateral molar clench restrained at the first molar (LUM1), peak 
$1^{\text {st }}$ principal stress values are seen around the postero-inferior proximal screw hole (Figure 3). This is also observed for the Von Mises and $3^{\text {rd }}$ principal stress plot; however, there is also notable Von Mises stress concentration in the postero-superior bone adjacent to the fracture plane that somewhat corresponds to the $3^{\text {rd }}$ principal stress plot in that region.

Observing the unilateral molar clenching tasks, all four experience peak bone stress for the three types of measures in the buccal cortical bone in the posterior bone section. For left side configurations (LUM1 and LUM2), the peak Von Mises, 1st principal stress, and $3^{\text {rd }}$ principal stress occur around the postero-inferior screw hole. In a similar fashion, all stress measures for the contralateral clenching tasks coincide about the proximal postero-superior screw hole. The difference in magnitudes for the four unilateral molar clenching tasks ranges from $16.8 \%$ to $21.6 \%$ for the compressive stress compared to the Von Mises stress. The ipsilateral bites produce larger Von Mises stress values, while the contralateral unilateral molar clenching tasks exhibit lesser values (86 MPa vs. $52 \mathrm{MPa}$ ). First principal stress values for all four unilateral molar clenching tasks range from approximately $64 \mathrm{MPa}$ to $73 \mathrm{MPa}$; with the right side biting tasks producing slightly larger values. The $3^{\text {rd }}$ principal stress ranged from -43 MPa to $-104 \mathrm{MPa}$ with the left side biting tasks producing greater compressive stress than the right side.

Canine clenching tasks consist of two tasks, ipsilateral (LUC) and contralateral (RUC) bites. The left unilateral canine clench showed a peak nodal bone Von Mises stress value of $43 \mathrm{MPa}$ at the postero-inferior proximal screw hole site and a maximum $1^{\text {st }}$ principal stress magnitude of $48 \mathrm{MPa}$ in the cortical bone surrounding the postero-superior screw hole in proximity to the fracture. The peak $3^{\text {rd }}$ principal stress of $-37 \mathrm{MPa}$ is noted at the proximal postero-inferior screw site region. On the other hand, the contralateral canine clench (RUC) demonstrated maximum Von Mises $(35 \mathrm{MPa}), 1^{\text {st }}$ principal stress $(44 \mathrm{MPa})$, and $3^{\text {rd }}$ principal stress $(-28.9 \mathrm{MPa})$ values at the proximal postero-superior hole site.

The intercuspal clench was restrained at each $2^{\text {nd }}$ premolar for the ICPM case and at the $1^{\text {st }}$ molars for the ICP1M case. For both clenching tasks the maximum peak nodal Von Mises stress and $3^{\text {rd }}$ principal stress values occur at the postero-inferior proximal screw-hole vicinity; whereas the maximum $1^{\text {st }}$ principal for both intercuspal cases occur at the postero-superior screw site. Similarly, the peak Von Mises, $1^{\text {st }}$ principal, and $3^{\text {rd }}$ principal stresses follow the same pattern for the bilateral molar clenches for both tooth restraint configurations.

$1^{\text {st }}$ principal stress maximums for the ipsilateral clenching tasks (LUM1 and LUM2) are located at the postero-inferior screw site, while all other clenching tasks peak values were found to be at the postero-superior screw region proximal to the fracture. With the exception of the contralateral clenching tasks, all other maximum compressive stress (minimum $3^{\text {rd }}$ principal stress) is found to be located at the proximal postero-inferior screw location. The contralateral biting tasks experience maximum compression around the proximal postero-superior screw cortical bone. The peak Von Mises stress values for the unilateral clench- 
ing tasks occur at the proximal postero-inferior screw region, except for the right unilateral molar clenches, these occur at the proximal postero-superior screw vicinity. With the exception of the incisal clenching task, whose peak Von Mises stress is found in the area of the proximal antero-superior screw hole, the remaining bilateral clenching tasks exhibit peak Von Mises stress value near the postero-inferior screw hole.

\subsubsection{Plate-Screw Von Mises Stress}

Plate and screw Von Mises stress measures were observed to determine the viability of the plates and screws to withstand the loading from the different bite loads. Plate Von Mises stress contour plots indicate, for all bilateral and ipsilateral clenching tasks, that maximum stress values are found at the inferior plate band bridging the fracture (Figure 4). Contralateral loading produces peak stress values across the fracture region in the superior plate band. The distal plate bands exhibit minimal loading for all bite cases. With the exception of the right unilateral canine clench, unilateral cases demonstrate higher peak Von Mises stresses than the bilateral clenching tasks. The range for the peak plate Von Mises stress values range from $285 \mathrm{MPa}$ to $504 \mathrm{MPa}$ (Table 6).

Peak screw Von Mises stress values for all clenching tasks are found in the screw locations proximal to the fracture. Bilateral molar clenching tasks exhibit peak Von Mises stress values in the proximal antero-inferior screw. The incisal clench shows a max value at the postero-superior screw location. Intercuspal peak stress value is found for the second premolar restraint in the antero-inferior screw while with the restraint at the $1^{\text {st }}$ molar gives a maximum at the antero-superior screw. For the unilateral canine clenching tasks, the left side bite (LUC) demonstrates a peak Von Mises stress value in the antero-superior screw while the right side bite (RUC) produces a maximum value in the postero-superior screw. Both right unilateral clenching tasks (RUM1 and RUM2) produce maximum screw Von Mises values at postero-superior region, while both left side clenching maximum screw stresses occur at the postero-inferior location. Referring to Table 6, peak von Mises stress in the screw bodies range from $89 \mathrm{MPa}$ to $118 \mathrm{MPa}$.
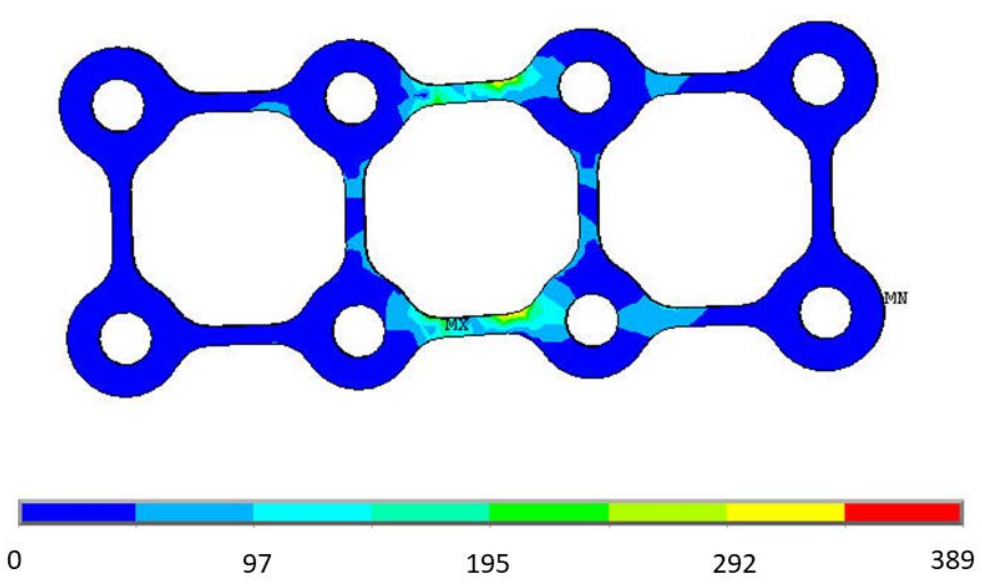

Figure 4. Plate Von Mises Stress (MPa) for an incisal clench (INC). 
Table 6. Plate and screw von mises (VM) stress for all biting tasks. Note: BM1: bilateral $1^{\text {st }}$ molar clench, BM2: bilateral $2^{\text {nd }}$ molar clench, ICPM: intercuspal clench at 2 nd premolar IC1M: intercuspal clench at $1^{\text {st }}$ molar, INC: Incisal, RUC: right unilateral canine clench, LUC: left unilateral canine clench, LUM1: left unilateral molar clench restrained at the 1st molar, LUM2: left unilateral molar clench restrained at the 2nd molar, RUM1: right unilateral molar clench restrained at the 1st molar, RUM2: right unilateral molar clench restrained at the $2^{\text {nd }}$ molar.

\begin{tabular}{ccc}
\hline Bite Task & Plate VM Stress $(\mathrm{MPa})$ & Screw VM Stress $(\mathrm{MPa})$ \\
\hline BM1 & 302 & 91 \\
BM2 & 295 & 93 \\
ICPM & 371 & 110 \\
IC1M & 400 & 118 \\
INC & 389 & 89 \\
RUC & 285 & 70 \\
LUC & 502 & 115 \\
LUM1 & 423 & 108 \\
LUM2 & 443 & 110 \\
RUM1 & 504 & 118 \\
RUM2 & 448 & 106 \\
\hline
\end{tabular}

\subsection{Displacement Results}

Relative displacement along the fracture provides insight into the behavior of the bone-hardware deformation behavior at the fracture site. The points of interest are at the superior and inferior nodes corresponding to the midpoint of the fracture plane. The relative displacement is measured at two coincident nodes on each side of the fracture. The $x$-axis is taken to be the transverse direction across the fracture, along the antero-posterior axis (see Figure 5). The $y$-axis is in the vertical direction parallel to the fracture plane. The $z$-axis is parallel to the fracture plane in the buccal-lingual direction and perpendicular to the $x$-axis. For illustration, displacement contour plots of $x, y, z$, and resultant magnitude displacements are respectively displayed in Figure 5 for a BM2 clench.

\subsubsection{Superior Nodal Relative Displacement}

The bilateral bite task that produced the greatest displacement along the $x$-axis is the ICPM bite task while the least displacement of the superior nodes is generated by the BM2 task (Table 7). The INC bite causes slightly more displacement than the IC1M clench, and the BM1 clench relative separation displacement in the $x$-direction is only $0.1 \%$ greater BM2 task. In general, the right unilateral clenching tasks produced greater relative displacement of the fracture in the horizontal direction than their corresponding left unilateral clenching counterparts. The LUM1 bite exhibits the least amount of $x$-direction displacement of the unilateral clinches while the greatest displacement in the transverse direction is generated by the RUM1 clench. Vertical translation relative displacement 
Bilateral Molar Clench at $2^{\text {nd }}$ Molar

X-Dișp: Normal to Fracture Plane (mm)
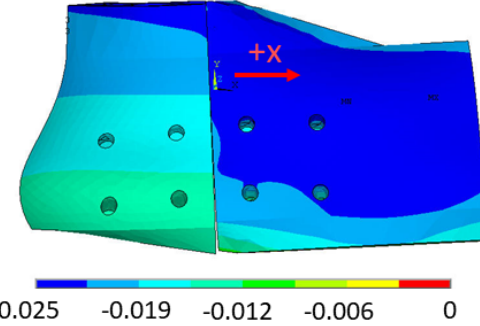

Y-Disp: Inf/Sup in Fracture Plane ( $\mathrm{mm})$

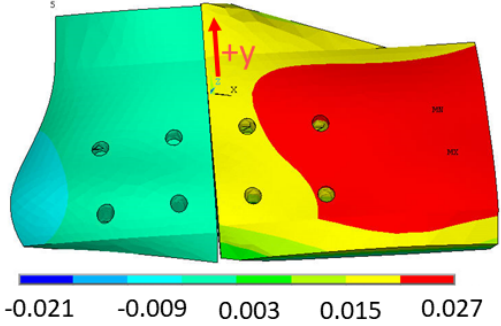

Z-Disp: Bucal(+)/Lingual Direction in Fracture Plane $(\mathrm{mm})$

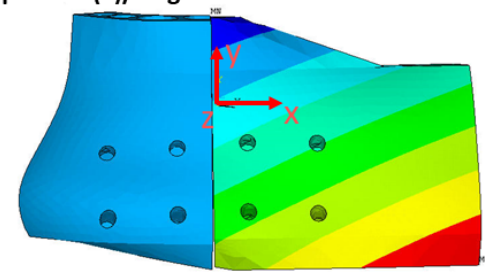

$\begin{array}{lllll}-0.031 & 0.003 & 0.037 & 0.071 & 0.106\end{array}$

Resultant Displacement (mm)

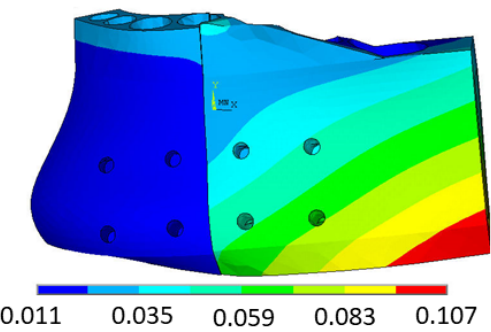

Figure 5. Displacement contour plot of $x, y, z$ and resultant displacement (mm) for bilateral molar clench at the $2^{\text {nd }}$ molar.

Table 7. Relative displacement in $\mathrm{mm}$ at fracture mid-plane superior nodes. Percentages are with respect to the minimum value in each column.

\begin{tabular}{|c|c|c|c|c|c|c|c|c|}
\hline Bite Task & $X$ & $Y$ & $Z$ & Resultant & $\% X$ & $\% Y$ & $\% Z$ & \%Resultant \\
\hline BM1 & 0.0043 & 0.0150 & 0.0226 & 0.0287 & 0.1 & 70.2 & 302.7 & 6.9 \\
\hline BM2 & 0.0043 & 0.0120 & 0.0240 & 0.0277 & 0.0 & 36.2 & 326.7 & 3.1 \\
\hline ICPM & 0.0172 & 0.0156 & 0.0110 & 0.0269 & 299.8 & 77.3 & 96.0 & 0.0 \\
\hline IC1M & 0.0055 & 0.0213 & 0.0287 & 0.0381 & 28.8 & 141.8 & 410.9 & 41.9 \\
\hline INC & 0.0059 & 0.0303 & 0.0210 & 0.0376 & 38.3 & 244.2 & 273.4 & 39.7 \\
\hline RUC & 0.0180 & 0.0297 & 0.0056 & 0.0353 & 318.6 & 237.2 & 0.0 & 31.3 \\
\hline LUC & 0.0153 & 0.0088 & 0.0728 & 0.0749 & 255.0 & 0.0 & 1195.7 & 178.8 \\
\hline LUM1 & 0.0084 & 0.0325 & 0.0282 & 0.0440 & 94.6 & 268.9 & 402.4 & 63.7 \\
\hline LUM2 & 0.0111 & 0.0409 & 0.0483 & 0.0645 & 159.0 & 364.7 & 759.7 & 140.0 \\
\hline RUM1 & 0.0220 & 0.0181 & 0.0610 & 0.0673 & 411.1 & 105.5 & 985.7 & 150.5 \\
\hline RUM2 & 0.0157 & 0.0508 & 0.0301 & 0.0611 & 265.1 & 476.4 & 435.9 & 127.3 \\
\hline
\end{tabular}

computations demonstrate the LUC clench causing the least amount of relative displacement. A RUM2 clenching bite results in the greatest relative translation along the $y$-axis for the superior nodes. The RUC bite provided for the least displacement in the $z$ direction while its counterpart task (LUC), resulted in the greatest $Z$-axis relative translation. The resultant relative magnitude displacement observation yields the least relative translation by the ICPM clenching task and the largest relative resultant displacement magnitude is given by the LUC biting task. 


\subsubsection{Inferior Nodal Relative Displacement}

The minimum translation in all directions at the inferior node occurs for the INC bite force (Table 8). The largest translation in $x$-axis is shown for the ICPM task, followed by the ipsilateral molar clenching tasks. Ipsilateral molar clenching tasks also generated the most relative translation along the vertical direction. Maximum relative displacement along the $z$-axis was produced by the LUM2 Tasks.

Table 9 averages the resultant relative translation produced by each clenching task. The INC task generates the smallest relative displacement while the LUM2 case produces the largest overall translation.

Table 8. Relative displacement $(\mathrm{mm})$ at inferior nodes percentages are with respect to values for the inciscal clinch (minimum value in each column).

\begin{tabular}{ccccccccc}
\hline Bite Task & $\mathrm{X}$ & $\mathrm{Y}$ & $\mathrm{Z}$ & Resultant & $\% \mathrm{X}$ & $\% \mathrm{Y}$ & $\% \mathrm{Z}$ & $\%$ Resultant \\
\hline BM1 & 0.0132 & 0.0130 & 0.0417 & 0.0456 & $265 \%$ & $427 \%$ & $135 \%$ & $149 \%$ \\
BM2 & 0.0129 & 0.0180 & 0.0423 & 0.0477 & $256 \%$ & $628 \%$ & $138 \%$ & $160 \%$ \\
ICPM & 0.0743 & 0.0229 & 0.0277 & 0.0825 & $1953 \%$ & $830 \%$ & $56 \%$ & $351 \%$ \\
IC1M & 0.0195 & 0.0140 & 0.0539 & 0.0590 & $439 \%$ & $467 \%$ & $203 \%$ & $22 \%$ \\
INC & 0.0036 & 0.0025 & 0.0178 & 0.0183 & $0 \%$ & $0 \%$ & $0 \%$ & $0 \%$ \\
nyyC & 0.0054 & 0.0248 & 0.0198 & 0.0322 & $50 \%$ & $907 \%$ & $12 \%$ & $76 \%$ \\
LUC & 0.0138 & 0.0078 & 0.0705 & 0.0722 & $282 \%$ & $217 \%$ & $296 \%$ & $294 \%$ \\
LUM1 & 0.0418 & 0.0683 & 0.0322 & 0.0863 & $1054 \%$ & $2670 \%$ & $81 \%$ & $371 \%$ \\
LUM2 & 0.0320 & 0.0511 & 0.0748 & 0.0961 & $785 \%$ & $1970 \%$ & $321 \%$ & $425 \%$ \\
RUM1 & 0.0084 & 0.0050 & 0.0589 & 0.0597 & $133 \%$ & $102 \%$ & $231 \%$ & $226 \%$ \\
RUM2 & 0.0070 & 0.0470 & 0.0202 & 0.0517 & $94 \%$ & $1807 \%$ & $14 \%$ & $182 \%$ \\
\hline
\end{tabular}

Table 9. Resultant comparison of inferior and superior node-percentages are with respect to the INC clench.

\begin{tabular}{ccccc}
\hline Bite Task & Resultant (Superior) & Resultant (Inferior) & Avg & \%Avg \\
\hline BM1 & 0.0287 & 0.0456 & 0.0372 & $33.1 \%$ \\
BM2 & 0.0277 & 0.0477 & 0.0377 & $34.9 \%$ \\
ICPM & 0.0269 & 0.0825 & 0.0547 & $95.8 \%$ \\
IC1M & 0.0381 & 0.0590 & 0.0486 & $73.8 \%$ \\
INC & 0.0376 & 0.0183 & 0.0279 & $0.0 \%$ \\
RUC & 0.0353 & 0.0322 & 0.0338 & $20.8 \%$ \\
LUC & 0.0749 & 0.0722 & 0.0736 & $163.3 \%$ \\
LUM1 & 0.0440 & 0.0863 & 0.0652 & $133.2 \%$ \\
LUM2 & 0.0645 & 0.0961 & 0.0803 & $187.4 \%$ \\
RUM1 & 0.0673 & 0.0597 & 0.0635 & $127.4 \%$ \\
RUM2 & 0.0611 & 0.0517 & 0.0564 & $101.8 \%$ \\
\hline
\end{tabular}




\section{Discussion}

\subsection{Stress}

\subsubsection{Bone Stress-Principal versus Von Mises}

There is a problem in the FEA literature on agreeing to what type of stress measure ought to be reported in studies of fracture fixation. Bone is characterized by many as a brittle material, which is often described as a material that is very resilient in compression but fails much more easily in tension and can endure very little strain. However, bone can fail in both tension and compression. Ultimate strength in tension ranges from $92-188 \mathrm{MPa}$ and ultimate strength in compression ranges from 133 to $295 \mathrm{MPa}$, depending on the mechanical test methods employed [19]. Von Mises stress is commonly considered most appropriate for ductile materials.

Many numerical studies of the mechanics of mandibular deformation using the finite element method have been carried out. However, there is still variation in reporting different stress measures in FEA studies of the mandible. Korioth et al. [20] exclusively report bone principal stresses in the study of the mechanical behavior a human mandible constructed from tomographs of a dry dentate mandible subject to functional bite forces. Comparisons of different fixation methods, following split ramus osteotomies, report all bone stress results using the Von Mises criteria [21] [22]. In simulations of the human mandible with and without an endosseous implant, Chen et al. [23] report stress values in principal, Von Mises and dilatational stress values. Simsek et al. [24] recognize that Von Mises and principal stresses seem to be reported equally in the literature. However, the authors note in their work that Von Mises is defined as the beginning of deformation for ductile materials and therefore opt to use the principal stress in reporting values. Kofod et al. [25] [26] simply report Von Mises in their study of the mandible and temporomandibular joint (TMJ) before and after distraction. Stress levels were calculated using the Von Mises stresses in an FEA study of the influence of occlusal loading on implants and supporting bone because FEA studies of this nature commonly tend to report the von Mises stress to characterize the state of stress in bone [27]. Tada et al. reported both stress measures but used the von Mises criteria since the scalar quantity is influenced by the principal stress components and thus facilitating the comparison of multiple models [28]. Other studies have utilized only the von Mises measure since it's the either the most prevalent in the literature or simply just reported qualitative stress distribution changes [6] [7] [9] [13] [27] [29].

This study found no conclusive direct correlation between the location of the peak Von Mises and principal stress values. There is one particular instance with the incisal clench that the maximum Von Mises was determined to be in the anterior bone segment while both peak tension and compressive values were located in the posterior bone region of the parasymphysis. Additionally, in many cases there was no direct correspondence of the von Mises stress location coinciding with location of peak principal stress values, in either tension or compres- 
sion. Since there is a possibility of failure of cortical bone in both tension and compression, and due to the complex nature of the geometry and loading and boundary conditions, it is conceivable for cortical bone to exhibit much higher compressive stresses than tensile as demonstrated by the left unilateral molar clenching tasks. In this particular study, all peak values for all tasks were well below the bone failure criteria. The observation of stress in tension or compression was most clearly distinguishable by the principal stress measures. The von Mises stress only calculates a magnitude of the state of stress at the point and may be subject to human misinterpretation. The reasons for reporting principal stress values in the literature and the insight gained from this study affirm the position that principal stress measures should be used in FEA mandibular bone studies as opposed to the von Mises criteria.

\subsubsection{Plate-Screw System}

For all biting tasks the plates are able to withstand the stress, even for the maximum plate stress of $502 \mathrm{MPa}$ for the ipsilateral canine clench (LUC), which is well below the yielding strength of the titanium, which is above $880 \mathrm{MPa}$. The screws experienced even less stress with a maximum of $118 \mathrm{MPa}$ for the right unilateral molar clench restrained at the first tooth (RUM1). This plate-system has previously been determined to demonstrate stability for unilateral clenching tasks at the first molar [7] and this study concludes that the fixation and fracture configuration is stable for other biting tasks. Since screws are bonded and cylindrical, this does not realistically model true screw geometry, although a previous study concluded that cylindrical and helical structured screws may have similar stress distributions [28].

\subsection{Bone Healing and Displacement}

Complete bone healing is the goal of internal fixation devices, and the stability provided by the fixation system is vital to facilitate the process. Failures include nonunion, malunion, malocclusion, infection and osteomyelitis, and hardware failures such as a broken plate or loosening and screw back-out. The primary goal of the procedure is to stabilize the fracture and allow an early return to function [30] [31]. For direct bone healing to occur, minimal deformation needs to exist such that osteons can form to link the two fragments together. If higher interfragmentary motion is observed, a secondary bone union process occurs via a callus formation to join the two fragments to form bone. Lovald et al. [7] reports an allowable range of $0.15 \mathrm{~mm}$, which all values in this study in any direction fall under. Expectedly, the ipsilateral biting tasks (left side in the model), produce the largest relative displacement and should be minimized during the initial stages of healing. The bilateral clenching tasks (BM1, BM2, ICPM, IC1M) produced a moderate amount of translation with the incisal clench (INC) producing the least amount. The greatest displacement is generated by the ipsilateral LUC and LUM2 clenching tasks. For all cases, loading on the contralateral side reduce fracture motion. 


\subsection{Boundary Conditions and Muscle Forces}

One of the greatest challenges in evaluating mandibular fracture FEA has been the determination of muscle forces boundary conditions [32]. Several studies, including this one, have fixed the condyles and have interpreted the results through a qualitative lens [6] [7] [13] [21] [22] [23] [33] [34]. Due to the complexities of the condyle, articular disk, and boundary conditions at the temporomandibular joints, the large majority of FEA studies have modeled only the mandible and completely fixed the condyles. Some attempts to incorporate the "buffer" effect of the articular disk are to bond the condyle, articular disk, and fix the glenoid fossa bone [14] [20]. This still limits the degree of freedom that the articular disk normally provides at the TMJ. The justification for this is based on St. Venant's Principle which states that stresses far away from a load are unaffected by the local stress at the point of loading. Another method is to represent the mandible without the articular disk and use "gap" elements that determine the boundary at which displacement is not to exceed; and used in conjunction with spring elements. This is probably a closer approximation but still does not take into account the friction encountered with rotation. For this treatment, the articular disk is assumed to behave in a uni-axial fashion and it is unclear as to what the contact interaction is with the glenoid fossa (i.e. friction, elastic properties) [25] [26] [35]. Yet another method is to apply spring elements on nodes fixed on a symmetry plane and compute reaction forces prior to applying the necessary balancing forces at the condyle [36]. The model may be underconstrained with this treatment.

Further concerns in modeling biting loads are the boundary conditions applied at the teeth. For the unilateral or incisal clenching tasks, the point of contact is only one tooth. This type of loading best represents some sort of small object (e.g. a peanut) such that the muscles are fully engaged at the initiation of mastication. The bilateral clenching tasks assume bite contacts which are symmetrical and bilateral with occlusal contact only occurring at two teeth. However, this still offers some qualitative inference for the patient that is simply clenching unconsciously while awake or asleep. There is no literature, to the author's knowledge that has taken into consideration the occlusal contact relationship with the upper teeth for any bite clench. This study is conservative in that fixation such as arch bars, or other devices which will limit mastication functioning, are not considered in this model.

\section{Conclusion}

The inclusion of multiple forces contributes to the understanding of the fixation device under multiple force loads allowing the identification potential failure due to patient non-compliance. Principal stress measures offer the best insight into the type of stress (i.e. tension or compression) bone is subjected to during loading. The finite element method is a capable tool for aiding in the analysis of mandibular fractures. Mandible studies can still benefit from refined screw/bone 
modeling and interaction. Furthermore, limitations in the number of elements and nodes imposed on academic research institutions to accurately refine the elements to conform to the geometrical CAD model influence the degree to which reality can be simulated, including screw/bone interaction. Significant progress has been made with the inclusion of contact, friction, and broadening the range of biting tasks in fracture models such that qualitative insight can be explored in conjunction with surgeons to interpret results based on their experiences with patients.

\section{Conflicts of Interest}

The authors declare no conflicts of interest regarding the publication of this paper.

\section{References}

[1] Hanson, J., Lovald, S., Cowgill, I., Erdman, M. and Diamond, B. (2011) National Hardware Removal Rate Associated with Internal Fixation of Facial Fractures. Journal of Oral and Maxillofacial Surgery, 69, 1152-1158. https://doi.org/10.1016/j.joms.2010.05.029

[2] Lamphier, J., Ziccardi, V., Ruvo, A. and Janel, M. (2003) Complications of Mandibular Fractures in an Urban Teaching Center. Journal of Oral and Maxillofacial Surgery, 61, 745-749. https://doi.org/10.1016/S0278-2391(03)00147-2

[3] Murthy, A.S. and Lehman, J.A. (2005) Symptomatic Plate Removal in Maxillofacial Trauma: A Review of 76 Cases. Annals of Plastic Surgery, 55, 603-607. https://doi.org/10.1097/01.sap.0000183802.38116.37

[4] Bhatt, V. and Langford, R.J. (2003) Removal of Miniplates in Maxillofacial Surgery: University Hospital Birmingham Experience. Journal of Oral and Maxillofacial Surgery, 61, 553-556. https://doi.org/10.1053/joms.2003.50108

[5] Kim, Y.K. and Nam, K.W. (2001) Treatment of Mandible Fractures Using Low-Profile Titanium Miniplates: Preliminary Study. Plastic and Reconstructive Surgery, 108, 38-43. https://doi.org/10.1097/00006534-200107000-00007

[6] Caraveo, V., Lovald, S., Khraishi, T., Wagner, J. and Baack, B. (2008) The Effects of Frictionless/Frictional Contact Boundary Conditions in Finite Element Modeling of Mandibular Fractures. Multidiscipline Modeling in Materials and Structures, 4, 227-236. https://doi.org/10.1163/157361108784890714

[7] Lovald, S.T., Khraishi, T., Wagner, J., Baack, B., Kelly, J. and Wood, J. (2006) Comparison of Plate-Screw Systems Used in Mandibular Fracture Reduction: Finite Element Analysis. Journal of Biomechanical Engineering, 128, 654-662. https://doi.org/10.1115/1.2244575

[8] Lovald, S.T., Wagner, J.D. and Baack, B. (2009) Biomechanical Optimization of Bone Plates Used in Rigid Fixation of Mandibular Fractures. Journal of Oral and Maxillofacial Surgery, 67, 973-985. https://doi.org/10.1016/j.joms.2008.12.032

[9] Chaudhary, N., Lovald, S., Wagner, J., Khraishi, T. and Baack, B. (2008) Experimental and Numerical Modeling of Screws Used for Rigid Internal Fixation of Mandibular Fractures. Modelling and Simulation in Engineering, 2008, Article ID: 628120. https://doi.org/10.1155/2008/628120

[10] Schwartz-Dabney, C.L. and Dechow, P.C. (2003) Variations in Cortical Material Properties throughout the Human Dentate Mandible. American Journal of Physical 
Anthropology, 120, 252-277. https://doi.org/10.1002/ajpa.10121

[11] Wade, A. and Wagner, J. (2003) A Retrospective Review of the Effect of Delayed versus Acute Treatment of Mandibular Fractures at a University Teaching Hospital. University of New Mexico, Albuquerque.

[12] Craig, R.G. and Peyton, F.A. (1958) Elastic and Mechanical Properties of Human Dentin. Journal of Dental Research, 37, 710-718. https://doi.org/10.1177/00220345580370041801

[13] Cox, T., Kohn, M.W. and Impelluso, T. (2003) Computerized Analysis of Resorbable Polymer Plates and Screws for the Rigid Fixation of Mandibular Angle Fractures. Journal of Oral and Maxillofacial Surgery, 61, 481-487. https://doi.org/10.1053/joms.2003.50094

[14] Korioth, T.W., Romilly, D.P. and Hannam, A.G. (1992) Three-Dimensional Finite Element Stress Analysis of the Dentate Human Mandible. American Journal of Physical Anthropology, 88, 69-96. https://doi.org/10.1002/ajpa.1330880107

[15] Shockey, J.S., von Fraunhofer, J.A. and Seligson, D. (1985) A Measurement of the Coefficient of Static Friction of Human Long Bones. Surface Technology, 25, 167-173. https://doi.org/10.1016/0376-4583(85)90030-5

[16] Nelson, G.J. (1986) Three Dimensional Computer Modeling of Human Mandibular Biomechanics. The University of British Columbia, Vancouver.

[17] Tate, G.S., Ellis, E. and Throckmorton, G. (1994) Bite Forces in Patients Treated for Mandibular Angle Fractures: Implications for Fixation Recommendations. Journal of Oral and Maxillofacial Surgery, 52, 734-736. https://doi.org/10.1016/0278-2391(94)90489-8

[18] Gray, H. (1995) Gray's Anatomy. 15th Edition, Elsevier, New York. https://doi.org/10.1016/S0260-6917(95)80090-5

[19] An, Y.H. and Draughn, R.A. (2000) Mechanical Testing of Bone and the Bone-Implant Interface. CRC Press, Boca Raton. https://doi.org/10.1201/9781420073560

[20] Korioth, T.W. and Versluis, A. (1997) Modeling the Mechanical Behavior of the Jaws and Their Related Structures by Finite Element (FE) Analysis. Critical Reviews in Oral Biology and Medicine, 8, 90-104. https://doi.org/10.1177/10454411970080010501

[21] Erkmen, E., Simsek, B., Yucel, E. and Kurt, A. (2005) Comparison of Different Fixation Methods Following Sagittal Split Ramus Osteotomies Using Three-Dimensional Finite Elements Analysis. Part 1: Advancement Surgery-Posterior Loading. International Journal of Oral and Maxillofacial Surgery, 34, 551-558. https://doi.org/10.1016/j.ijom.2004.10.009

[22] Erkmen, E., Simsek, B., Yucel, E. and Kurt, A. (2005) Three-Dimensional Finite Element Analysis Used to Compare Methods of Fixation after Sagittal Split Ramus Osteotomy: Setback Surgery-Posterior Loading. The British Journal of Oral \& Maxillofacial Surgery, 43, 97-104. https://doi.org/10.1016/j.bjoms.2004.10.007

[23] Chen, J., Lu, X., Paydar, N., Akay, H.U. and Roberts, W.E. (1994) Mechanical Simulation of the Human Mandible with and without an Endosseous Implant. Medical Engineering \& Physics, 16, 53-61. https://doi.org/10.1016/1350-4533(94)90011-6

[24] Simsek, B., Erkmen, E., Yilmaz, D. and Eser, A. (2006) Effects of Different Inter-Implant Distances on the Stress Distribution around Endosseous Implants in Posterior Mandible: A 3D Finite Element Analysis. Medical Engineering \& Physics, 28, 199-213. https://doi.org/10.1016/j.medengphy.2005.04.025

[25] Kofod, T., Cattaneo, P.M., Dalstra, M. and Melsen, B. (2005) Three-Dimensional 
Finite Element Analysis of the Mandible and Temporomandibular Joint during Vertical Ramus Elongation by Distraction Osteogenesis. The Journal of Craniofacial Surgery, 16, 586-593. https://doi.org/10.1097/01.SCS.0000157305.60505.B5

[26] Kofod, T., Cattaneo, P.M. and Melsen, B. (2005) Three-Dimensional Finite Element Analysis of the Mandible and Temporomandibular Joint on Simulated Occlusal Forces before and after Vertical Ramus Elongation by Distraction Osteogenesis. The Journal of Craniofacial Surgery, 16, 421-429. https://doi.org/10.1097/01.scs.0000171969.32110.ab

[27] Eskitascioglu, G., Usumez, A., Sevimay, M., Soykan, E. and Unsal, E. (2004) The Influence of Occlusal Loading Location on Stresses Transferred to Implant-Supported Prostheses and Supporting Bone: A Three-Dimensional Finite Element Study. The Journal of Prosthetic Dentistry, 91, 144-150. https://doi.org/10.1016/j.prosdent.2003.10.018

[28] Tada, S., Stegaroiu, R., Kitamura, E., Miyakawa, O. and Kusakari, H. (2003) Influence of Implant Design and Bone Quality on Stress/Strain Distribution in Bone around Implants: A 3-Dimensional Finite Element Analysis. The International Journal of Oral \& Maxillofacial Implants, 18, 357-368.

[29] Chuong, C.J., Borotikar, B., Schwartz-Dabney, C. and Sinn, D.P. (2005) Mechanical Characteristics of the Mandible after Bilateral Sagittal Split Ramus Osteotomy: Comparing 2 Different Fixation Techniques. Journal of Oral and Maxillofacial Surgery, 63, 68-76. https://doi.org/10.1016/j.joms.2003.12.045

[30] Schilli, W. (1998) Mandibular Fractures. In: Prein, J., Ed., Manual of Internal Fixation in the Cranio-Facial Skeleton, Springer Verlag, Berlin, 57-93. https://doi.org/10.1007/978-3-642-58789-4_3

[31] Mathog, R.H., Toma, V., Clayman, L. and Wolf, S. (2000) Nonunion of the Mandible: An Analysis of Contributing Factors. Journal of Oral and Maxillofacial Surgery, 58, 746-752. https://doi.org/10.1053/joms.2000.7258

[32] Daegling, D.J. and Hylander, W.L. (2000) Experimental Observation, Theoretical Models, and Biomechanical Inference in the Study of Mandibular Form. American Journal of Physical Anthropology, 112, 541-551. https://doi.org/10.1002/1096-8644(200008)112:4<541::AID-AJPA8>3.0.CO;2-Z

[33] Fernandez, J.R., Gallas, M., Burguera, M. and Viano, J.M. (2003) A Three-Dimensional Numerical Simulation of Mandible Fracture Reduction with Screwed Miniplates. Journal of Biomechanics, 36, 329-337. https://doi.org/10.1016/S0021-9290(02)00416-5

[34] Marinescu, R., Daegling, D.J. and Rapoff, A.J. (2005) Finite-Element Modeling of the Anthropoid Mandible: The Effects of Altered Boundary Conditions. The Anatomical Record Part A, Discoveries in Molecular, Cellular, and Evolutionary Biolo$g y$, 283, 300-309. https://doi.org/10.1002/ar.a.20166

[35] Castano, M.C., Zapata, U., Pedroza, A., Jaramillo, J.D. and Roldan, S. (2002) Creation of a Three-Dimensional Model of the Mandible and the TMJ in Vivo by Means of the Finite Element Method. International Journal of Computerized Dentistry, $\mathbf{5}$, 87-99.

[36] Choi, A.H., Ben-Nissan, B. and Conway, R.C. (2005) Three-Dimensional Modelling and Finite Element Analysis of the Human Mandible during Clenching. Australian Dental Journal, 50, 42-48. https://doi.org/10.1111/j.1834-7819.2005.tb00084.x 